Fabiana M . de Andrade e M ara H. Hutz 1

\section{0 componente genético da determinação dos lipídeos séricos}

\author{
The genetic component of serum \\ lipid determination
}

Abstract Serum lipid levels are multifactorial traits determined by a high number of genetic and environmental factors. The identification of the genetic component of this traits have been intensively investigated in the last years. These studies focused mainly on polymorphisms in genes coding for structural proteins and enzymes related to lipid metabolism. M ore recent studies showed that the effect of these polymorphisms depend in part on the interactions of different genotypes with classic risk factors like tabagism, overweight or physical inactivity. The variability observed in these genes seems to influence drug response of those commonly used in hyperlipidemia treatment.

Key words M ultifactorial features, Lipid levels, Polymorphisms
Resumo O s níveis de lipídeos séricos são características multifatoriais determinadas por um grande número de fatores genéticos e ambientais. A identificação do componente genético dessas características tem sido intensamente investigada nos últimos anos. Esses estudos têm enfocado principalmente polimorfismos nos genes que codificam proteínas estruturais e enzimas relacionadas com o metabolismo de lipídeos. Estudos mais recentes mostraram que 0 efeito desses polimorfismos depende em parte das interações dos diferentes genótipos com os fatores de risco clássicos tais como tabagismo, sobrepeso ou sedentarismo. A variabilidade encontrada nesses genes parece também influir na resposta a fármacos comumente utilizados no tratamento das hiperlipidemias.

Palavras-chave Características multifatoriais, N íveis lipídicos, Polimorfismos
1 Departamento de Genética, Instituto de Biociências, Universidade Federal do Rio Grande do Sul. Caixa Postal 15.053 91501-970 Porto Alegre RS fabiana.andrade@ufrgs.br 


\section{Introdução}

\section{Epidemiologia}

A aterosclerose é a principal causa de morte em todo o mundo (M urray \& Lopes, 1997). Dados do Sistema de Informações sobre M ortalidade do SU S (DATA SUS, 1998) mostram que em 1998 a proporção de mortes decorrentes da aterosclerose no Brasil foi de $6.2 \%$.

As doenças isquêmicas do coração são causadas por uma grande variedade de fatores. Dentre estes, os mais amplamente relacionados com o referido grupo de patologias são os níveis séricos de lipídeos e lipoproteínas (revisão em M aron et al., 1998). Níveis elevados de LD L e triglicerídeos representam um fator de risco (Hokanson \& Austin, 1996), enquanto um grande número de estudos epidemiológicos relaciona baixos níveis de HDL como preditores, ealtos níveis desta lipoproteína como protetores para as cardiopatias (Goldbourt et al., 1997). Foi estimado que um decréscimo de $10 \%$ nos níveis de colesterol total em uma população pode resultar em redução de $30 \%$ na incidência de cardiopatias (American H eart Association, 2001).

Em uma amostra do sul do Brasil (M. H. Hutz, dados não publicados), $42 \%$ dos indivíduos apresentam níveis de colesterol total acima do desejável $(200 \mathrm{mg} / \mathrm{dL})$ de acordo com normas da NCEP (2001), enquanto $17 \%$ estão no grupo de risco (colesterol total acima de $240 \mathrm{mg} / \mathrm{dL}$ ). Além disso, 35\% têm níveis de HDL inferiores aos recomendados, e em $20 \%$ registram-se níveis de triglicerídeos superiores a $180 \mathrm{mg} / \mathrm{dL}$. U m perfil lipídico de risco parece ser uma característica extremamente comum em nossa população.

\section{Genética e características complexas: os níveis lipídicos}

N os últimos 15 anos, avanços sem precedentes têm sido alcançados no campo da genética das doenças multifatoriais, tais como cardiopatias, diabetes, obesidade e câncer. Desde 1982 mais de 20 genes codificantes para proténas envolvidas no transporte de lipídeos foram identificados, localizados cromossomicamente, e tiveram suas seqüências de DN A caracterizadas (Dammerman \& Breslow 1995). 0 anúncio oficial da conclusão do Projeto Genoma H umano, em fevereiro deste ano (International $\mathrm{Hu}$ man Genome Sequencing Consortium, 2001), representou um marco histórico. Esta proeza é considerada um importante passo no objetivo de revelar as bases genéticas de doenças complexas (Humphries \& Ordovas, 2001).

A variação dos níveis lipídicos é uma característica de etiologia multifatorial, pois é determinada por uma ampla gama de fatores, tanto ambientais quanto genéticos. Variações em um grande número de genes envolvidos na síntese de proteínas estruturais e enzimas relacionadas no metabolismo de lipídeos poderiam, a princípio, responder por variações do perfil lipídico de cada indivíduo. Desta maneira, qualquer gene que seja responsável pela produção de uma proteína envolvida nesta rota metabólica poderia ser um "gene candidato" na investigação de determinantes genéticos dos níveis lipídicos. Assim, o somatório de variações com pequeno efeito em cada um destes genes poderia levar à deterioração do perfil lipídico de um indivíduo, predispondo à cardiopatia. Como estas variantes genéticas são bastante freqüentes na população em geral (de $1 \%$ a $80 \%$ dos indivíduos, de acordo com exemplos na tabela 1), seu impacto é muito maior na saúde pública quando comparadas com mutações de grande efeito, mas que são muito mais raras.

\section{Variantes alélicas}

U ma área promissora da genética é a investigação de variações no DNA entre indivíduos. Estas variações são chamadas polimorfismos quando são freqüentes ( mais de $1 \%$ de freqüência do alelo mais raro). A base genética para esta variação pode ser uma troca de bases no DNA, uma duplicação ou deleção de um ou vários pares de bases. Estimativas atuais sugerem que variações de uma única base entre indivíduos (single nucleotide polymorphisms, ou SN Ps) ocorrem na freqüência de 1 SN P a cada 1.300pb, ou seja, existem mais de 1.4 milhão de polimorfismos de substituição de uma única base em nosso genoma (International H uman Genome Sequencing Consortium, 2001).

\section{Variações alélicas e dislipidemias}

\section{Genes que influenciam os níveis de LDL e colesterol total}

Lipoproteínas de baixa densidade (LDLs) constituem $70 \%$ do colesterol total, e só são capazes de circular no plasma por conterem uma 
Tabela 1

Genes relacionados com níveis lipídicos.

\begin{tabular}{|c|c|c|c|c|}
\hline Gene & Cromossomo & $\begin{array}{l}\text { Alelo }(\mathbf{s}) \\
\text { relacionado (s) }{ }^{1}\end{array}$ & Freqüência2 & $\begin{array}{l}\text { Porção lipídica } \\
\text { modificada }\end{array}$ \\
\hline APOE & 19 & $\begin{array}{l}E 2 \downarrow \\
E 4 \uparrow\end{array}$ & $\begin{array}{r}\sim 8 \% \\
\sim 15 \%\end{array}$ & \multirow[t]{4}{*}{ Colesterol total e LDL } \\
\hline LDLR - Pvu II & 19 & $\mathrm{P}-\uparrow$ & $\sim 20 \%$ & \\
\hline APOB - Xba I & 2 & $x+\uparrow$ & $\sim 40 \%$ & \\
\hline APOB - ins/del3 & 2 & del $\uparrow$ & $\sim 35 \%$ & \\
\hline LPL - Hind III & 8 & $H+\uparrow$ & $\sim 65 \%$ & \multirow[t]{7}{*}{ triglicerídeos } \\
\hline LPL - Pvu II & 8 & $P+\uparrow$ & $\sim 50 \%$ & \\
\hline LPL - D9N & 8 & $N 9 \uparrow$ & $\sim 3 \%$ & \\
\hline LPL - S291N & 8 & $\mathrm{~S} 291 \uparrow$ & $\sim 4 \%$ & \\
\hline LPL - S447X & 8 & $X 447 \downarrow$ & $\sim 20 \%$ & \\
\hline APOCIII-SacI & 11 & $S+\uparrow$ & $12 \%$ & \\
\hline APOE & 19 & $\mathrm{E} 2 \downarrow(?)$ & $\sim 8 \%$ & \\
\hline & & & & \multirow{4}{*}{ HDL } \\
\hline APOAI & 11 & $\begin{array}{r}-75 \mathrm{a} \uparrow \\
\mathrm{M}-\uparrow\end{array}$ & $\begin{array}{r}\sim 20 \% \\
\sim 4 \% \\
\text { (continua) }\end{array}$ & \\
\hline CETP & 16 & $T+\downarrow$ & $\sim 40 \%$ & \\
\hline $\mathrm{HL}$ & 15 & $-514 c \downarrow$ & $\sim 80 \%$ & \\
\hline
\end{tabular}

$1 \uparrow$ relação com aumento dos níveis; $\downarrow$ relação com diminuição dos níveis; + denota presença do sítio de restrição, enquanto - denota ausência do sítio

2 M édias aproximadas das freqüências alélicas dentre várias populações (ver referências no texto)

3 ins denota alelo de inserção e del alelo de deleção.

? denota resultados não esclarecidos (ver texto)

porção protéica constituída principalmente pela apolipoproteína B (apo B). Estas partículas são formadas pela ação da lipoproteína lípase $(L P L)$, enzima cuja ação é modulada pelas apolipoproteínas (apos) C-I, C-II e C-III e E. A maneira pela qual a LDL chega ao seu destino, ou seja, células de tecidos periféricos, é através da ligação de alta afinidade de sua apo B ao receptor de LDL (LDLR).

U m gene que exerce uma forte influência sobre os níveis de LDL é o gene da apo $E$. Três diferentes seqüências, com freqüências polimórficas, que produzem duas trocas de aminoácidos foram identificadas no loco da apo $E$, codificando três alelos denominados E2, E3 e E4. U m grande número de investigações realizadas permite afirmar que a presença do alelo E4 determina o aumento dos níveis de LDL e colesterol total, enquanto o alelo E2, ao contrário, determina uma diminuição destes níveis (M ahley et al., 1995; Boer et al., 1997; FrikkeSchmidt et al., 2000). A única investigação realizada em uma população brasileira confirmou o efeito protetor do alelo E2, com relação aos níveis de colesterol total (de Andrade et al., 2000).
Polimorfismos nos locos da apo B (Kammerer et al., 1996; Korhonen et al., 1999; M achado et al., 2001) eLDLR (H umphries et al., 1991; Gudnason et al., 1999) têm sido sistematicamente associados à variabilidade nos níveis de LDL. Estas associações também foram identificadas em populações brasileiras por M attevi (2000) e Salazar et al. (2000). As variantes destes locos que parecem influenciar níveis de colesterol total eLDL podem ser observadas na tabela 1.

\section{Genes que influenciam os níveis detriglicerídeos}

O catabolismo de lipoproteínas ricas em triglicerídeos começa com a atividade da lipoproteína lipase (LPL), que necessita da ativação pela apolipoproteína CII. A atividade desta enzima é também modulada pela apo CIII, que exerce uma ação inibitória sobre a LPL e pela apolipoproteína E, quealtera a atividade da LPL de diferentes maneiras de acordo com a isoforma (E2, E3 ou E4) (Davignon et al., 1998).

Exemplos de variantes associadas aos níveis de triglicerídeos são apresentados na tabela 1. 
0 gene da LPL vem sendo exaustivamente investigado, principalmente os polimorfismos que resultam em três variantes funcionais (D 9N, S291N, S447X) e duas em introns ( $H$ ind III $e$ Pvu II). De uma forma geral essas variantes estão associadas ao aumento dos níveis de triglicerídeos, mas a mutação S447X, que determina uma proteína truncada devido à perda dos últimos dois aminoácidos da cadeia polipeptídica, parece determinar, em média, níveis mais baixos de triglicerídeos (H okanson, 1999; Wilttrup et al., 1999; Razzaghi et al., 2000).

$A$ variação alélica da apo $E$ também tem sido investigada com relação a este parâmetro. Embora já tenha sido realizado um grande número de estudos, ainda não está claro qual dos alelos da apo E pode estar associado ao aumento dos níveis de triglicerídeos. Algumas investigações detectaram 0 aumento destes níveis relacionados ao alelo E2 (Sephernia et al., 1989), ao alelo E4 (M ahley et al., 1995; Gerdes et al., 1996), e a ambos os alel os, onde somente homozigotos E3/E3 tinham uma tendência em níveis normais (Kamboh et al., 1995; Boer et al., 1997). Em uma investigação de uma amostra de brasileiros, o alelo E2 foi associado à diminuição destes níveis, enquanto o alelo E4 foi relacionado com o aumento (de Andrade et al., 2000). Polimorfismos no gene da apo C-III parecem também influenciar os níveis deste lipídeo (Ko et al., 1997; Dallongeville et al., 2000).

\section{Genes que influenciam os níveis de HDL}

O primeiro passo na formação desta lipoproteína é a liberação de lipídeos do meio intracelular para o meio externo, através de um receptor recentemente descoberto (BrooksWilson et al., 1999), denominado ABCA1 (ATP binding cassete protein type $A 1$ ). Estes lipídeos se ligam à apo Al, e então, o HDL nascente sofre a ação de diferentes enzimas como a proteína transferidora de ésteres de colesterol (CETP) e a lipase hepática (HL). U ma das maneiras pela qual os lipídeos são removidos do HDL acontece através de um receptor descrito em 1996 (Acton et al., 1996), denominado SRBI (scavenger receptor class $B$ type I).

Alguns estudos têm revelado que fatores genéticos representam um papel de $40 \%$ a $70 \%$ na determinação da concentração de HDL (Heller et al., 1993; Bu et al., 1994). Ainda assim, poucos estudos têm sido realizados nesta área com o objetivo de se determinar exatamente quais os genes responsáveis por esta modula- ção. $O$ pequeno número de investigações aponta, no entanto, para genes como os da apo Al, CETP, lipase hepática, e os receptores ABCA 1 e SRBI.

Dois polimorfismos na região promotora do gene da apo Al (posições - 75 e +83) parecem estar relacionados com o nível de produção da proteína (Wang et al., 1998). Ambas as variantes são identificadas pela enzima de restrição M sp I, são denominadas de a - $75 \mathrm{~g}$ e M sp I +/-, e vêm sendo relacionadas à variação nos níveis de HDL por alguns autores (W ang et al., 1996).

Variações nos genes da CETP (Corbex et al., 2000; Kark et al., 2000) e lipase hepática (HL) (Couture et al., 2000; Juo et al., 2001; Zambon et al., 2001) também vêm sendo associadas aos níveis de HDL. Recentemente, foram identificados al guns polimorfismos nos genes dos receptores de HDL, ABCA 1 e SRBI (Wang et al., 2000 e Acton et al., 1999, respectivamente). Poucas investigações sobre a relação destes polimorfismos com níveis de HDL foram realizadas até o momento, sem resultados conclusivos. U m resumo dos princi pais resultados referentes ao HDL encontra-se na tabela 1.

\section{Interações entre genes}

Embora o efeito de vários genes sobre o metabolismo de lipídeos tenha sido verificado até o momento, é crucial ter em mente que nenhum gene atua isoladamente sobre uma característica. Q uando a etiologia da característica é poligênica, o resultado no indivíduo é um somatório de pequenos efeitos de vários genes. Este tipo de determinação genética é a realidade para a quase totalidade dos traços com al gum componente genético.

No entanto, a análise deste tipo de característica se torna mais complexa, quando se leva em conta que a maior parte dos indivíduos apresentará alguns alelos responsáveis pelo aumento dos níveis de LDL, por exemplo, e outros alelos que terão o efeito de redução destes níveis. Deste modo, al gumas investigações faIharam em detectar a associação de marcadores genéticos com níveis lipídicos, mas quando esta análise foi realizada em subgrupos de alelos de outros genes, uma forte interação foi observada. Pedersen \& Berg (1990) investigaram o efeito do polimorfismo Pvu II no gene LDLR, e a variação no gene da apo $E$, sobre os níveis lipídicos. 0 efeito de aumento de colesterol total 
relacionado ao alelo $E^{*} 4$ somente foi detectado em indivíduos que não eram portadores do alelo P - do gene LDLR. U ma investigação realizada em uma amostra de brasileiros detectou a associação de uma variante do gene da apo C-I com LDL e colesterol total apenas em indivíduos homozigotos para apo E3 (Fiegenbaum, 2001). Além disso, é importante lembrar que genes envolvidos em outras rotas metabólicas relacionadas podem estar interagindo com 0 metabolismo de lipídeos, como foi demonstrado com variações no gene do receptor de estrógenos em brasileiros (Almeida, 2001).

\section{Interações entre genes e fatores ambientais}

Níveis de lipídeos e lipoproteínas não são características apenas poligênicas, mas, por serem altamente influenciadas por fatores ambientais, são traços de origem multifatorial. U m indivíduo pode ter uma combinação de alelos que esteja relacionada com a deterioração de seu perfil lipídico, mas se ele mantiver hábitos de vida saudáveis, a chance de ter seus níveis alterados diminui.

0 efeito de alguns polimorfismos, no entanto, só se manifestará em um contexto dependente de variáveis ambientais que também predisponham à dislipidemia.

O polimorfismo Taq I do gene da CETP nos mostra alguns bons exemplos. Vohl et al. (1999) registrou uma diminuição dos níveis de HDL associado ao alelo $T+$ somente em indivíduos com sobrepeso. Por outro lado, 0 aumento destes níveis foi associado ao alelo $T$ - apenas em indivíduos não-fumantes e sem excesso de peso (Freeman et al., 1994). Outras interações foram também observadas em brasileiros por Fiegenbaum (2001), como a interação de polimorfismos nos genes da apo C-I e apo C-III e tabagismo sobre níveis de triglicerídeos e HDL.

\section{Polimorfismos genéticos e a resposta a medicamentos}

É de conhecimento geral que quando vários pacientes recebem a mesma dosagem de uma droga em particular, o efeito pode ser eficaz na maioria, ter pouca ou nenhuma conseqüência para outros, ou ainda ser tóxico ou até mesmo fatal para um terceiro subconjunto. A área que se dedica ao estudo da variabilidade na respos- ta às drogas devido à hereditariedade é denominada de farmacogenética (N ebert, 1999).

Da mesma maneira que ocorre a interação entre genes e a interação gene/ambiente, a ação conjunta de diferentes polimorfismos e o uso de alguns medicamentos já foi observada por al guns autores. As interações de diferentes medicamentos hipolipemiantes e o polimorfismo do gene da apo $\mathrm{E}$ foram revisadas por $\mathrm{H}$ agberg et al. (2000). N os grupos de pacientes tratados com estatinas e gemfibrozil, a melhor resposta ao medicamento, ou seja, maior redução de níveis de colesterol total, LDL e triglicerídeos foi obtida em indivíduos portadores dos alelos E2 ou E3. Por outro lado, pacientes portadores do alelo E4 tratados com probucol têm aumentada a resposta ao medicamento, em comparação com portadores dos outros genótipos da APOE.

Outros polimorfimos em vários genes, especialmente os genes da CETP e lipase hepática, vêm sendo investigados com relação à resposta a diferentes tratamentos. Zambon et al. (2001) detectaram um maior aumento de HDL em pacientes portadores do al elo -514c da lipase hepática, quando tratados com estatinas, colestipol ou niacina.

\section{Perspectivas futuras}

De acordo com o que foi discutido nesta revisão, consideráveis progressos têm sido feitos no entendimento das bases genéticas do metabolismo de lipídeos. Contudo, mais investigações ainda são necessárias para outros genes não discutidos neste artigo, especial mente os que foram recentemente identificados. Além disso, embora vários locos tenham seu papel bem de finido nesta rota metabólica, estudos sobre as interações entre estes locos, e destes com fatores ambientais são imprescindíveis. Como foi ressaltado por W inkel man \& H ager (2000), 0 conhecimento atual da variação no genoma de nossa espécie pode ser comparado à fotografia por satélite de uma cidade: uma ferramenta com alto grau de resolução, mas incapaz de entender o funcionamento completo desta cidade. Portanto, muita pesquisa ainda é necessária neste campo.

Quando se fala de doenças com al gum componente genético, a reação típica de profissionais de saúde é de que a genética inserida no model o clássico de saúde pública não funciona, uma vez que os genótipos dos indivíduos não são modificáveis. De acordo com esta vi- 
são, a prevenção poderia levar a conseqüências eugênicas, ao impedir o nascimento de pessoas com genótipos específicos. M as para que a genética e a saúde pública possam interagir com sucesso, a comunidade médica precisa mudar 0 paradigma clássico de prevenção de doenças genéticas, e para que esta mudança ocorra, é necessário que mude primeiramente a percepção do conceito de doença genética (discutido em Khoury, 1997).

Exceto para a maior parte dos casos de traumas, praticamente todas as doen ças apresentam algum componente genético. 0 papel da hereditariedade nas doenças não é, por si mesmo, uma revelação nova. M as no passado, era considerado improvável que alguma coisa pudesse ser feita com esta informação, exceto guiar a vigilância médica baseada na história familiar (Collins \& M cKusik, 2001).

$N$ este ponto, uma questão é aberta: o que significa prevenção no contexto de doenças genéticas? O bviamente, a prevenção primária se refere à prevenção da doença para a qual o genótipo é um componente importante, como a maior parte das características multifatoriais. N estes casos, a preven ção não se refere, ou não deve se referir à prevenção de genótipos, isto é, prevenção de nascimentos de indivíduos com genótipos específicos. Alternativamente, estes cuidados se referem a interromper os co-fatores ambientais que interagem com os genótipos de susceptibilidade. Este tipo de prevenção abrange desde a modificação de hábitos de vi- da, que já seriam deletérios por si só, mas que somados ao perfil genético são mais perigosos, como o tabagismo, até a utilização do medicamento mais adequado para cada tipo genotípico. Collins \& M cKusick (2001) acreditam que, por volta de 2010, testes genéticos preditivos estarão disponíveis para várias doenças comuns, permitindo aos indivíduos que desejam esta informação tomarem decisões para reduzir os riscos de desenvolvimento da doença. $\mathrm{Pa}$ ra estes autores, por volta de 2020, o impacto da genética sobre a medicina será ainda mais amplo. A abordagem farmacogenética para prever a resposta aos medicamentos será a prática padrão para um bom número de doenças.

O bviamente, nossa sociedade terá que triIhar um longo caminho até ver estas previsões realizadas. Muitas investigações sobre as interações entre genes e destes com o ambiente ainda são necessárias. Além disso, a comunidade científica e médica necessita de dados de prevalência de alelos críticos em cada população e grupo étnico, para poder acessar o impacto destes alelos na saúde pública de cada grupo. $M$ as a ciência não pode andar sozinha. É imprescindível que a sociedade valorize e exija avan ços nas áreas de ética e legisl ação, e suas aplicações, com a finalidade de impedir o mau uso deste conhecimento. Somente deste modo, as contribuições da genética para a epidemiologia tornarão possível a aplicação deste conhecimento na prática da medicina e da saúde pública do século 21.

\section{Referências bibliográficas}

Acton S et al. 1996. Identification of scavenger receptor $\mathrm{SR}-\mathrm{BI}$ as a high density lipoprotein receptor. Science 271:518-520.

Acton S et al. 1999. Association of polymorphisms at the SR-BI gene locus with plasma lipid levels and body mass index in a white population. Arteriosclerosis, Thrombosis, and Vascular Biology 19:1.734-1.743.

Almeida S 2001. Análise da influência de polimorfimos no gene do receptor de estrógenos sobre os níveis lipídicos e aterosclerose na população de Porto Alegre. Dissertação de M estrado. Programa de Pós-Graduação em Genética e Biologia M olecular. UFRGS. Porto Alegre, $94 \mathrm{pp}$.

American H eart Association 2001. Heart and Stroke Statistical U pdate. Dallas, Texas: American H eart Association, 2000. http://www.americanheart.org 
Boer JM A et al.1997. Interactions between lifestyle-related factors and the apoE polymorphism on plasma lipids and apolipoproteins - the EARS study. Arteriosclerosis, Thrombosis, and Vascular Biology 17:1.6751.681.

Brooks-Wilson A et al. 1999. M utations in ABC1 in Tangier disease and familial high-density lipoprotein deficiency. Nature Genetics 22:336-345.

Bu X et al. 1994. Linkage analysis of the genetic determinants of high density lipoprotein concentrations and composition: evidence for involvement of the apolipoprotin A-II and cholesteryl ester transfer protein loci. Human Genetics 93:639-648.

Collins FS \& M cKusick VA 2001. Implications of the human genome project for medical science. Journal of American M edical Association 285:540-544.

Corbex $\mathrm{M}$ et al. 2000. Extensive association analysis between the CETP gene and coronary heart disease phenotypes reveals several putative functional polymorphisms and gene-environment interaction. Genetic Epidemiology 19:64-80.

Couture $P$ et al. 2000. Association of the c-514t polymorphism in the hepatic lipase gene with variations in lipoprotein subclass profiles - the Framingham Offspring Study. Arteriosclerosis, Thrombosis, and Vascular Biology 20:815-822.

DallongevilleJ et al. 2000. Gender related association between genetic variations of APOC-III gene and lipid and lipoprotein variables in northern France. Atherosclerosis 150:149-157.

Dammerman M \& Breslow JI 1995. Genetic basis of lipoprotein disordens. Circulation 91:505-512.

DATA SUS: Informações de saúde. M ortalidade (1998). http://www.datasus.gov.br/

Davignon J, Gregg RE \& Sing CF 1988. A polipoprotein E polymorphism and atherosclerosis. Arteriosclerosis 8:1-21.

de Andrade FM , Larrandaburu M, Callegari-Jacques SM , Gastaldo G \& Hutz MH 2000. Association of apolipoprotein E polymorphism with plasma lipids and Alzheimer's disease in a Southern Brazilian population. Brazilian Journal of $\mathrm{M}$ edical and Biological Research 33:529-537.

Fiegenbaum M 2001. Estudo da variabilidade em genes de apolipoproteínas sobre níveis lipídicos e parâmetros de massa e gordura corporal na população de Porto Alegre. Dissertação de M estrado. Programa de Pós-Graduação em Genética e Biologia M olecular, UFRGS, Porto Alegre. $95 \mathrm{pp}$.

Freeman DJ et al. 1994. Regulation of plasma HDL cholesterol and subfraction distribution by genetic and environmental factors - associations between the TaqIB RFLP in the CETP gene and smoking and obesity. Arteriosclerosis and Thrombosis 14:336-344.

Frikke-Schmidt R, N ordestgaard BG, A gerholm-Larsen B, Schnohr P \& Tybjærg-H ansen A 2000. Contextdependent and invariant associations between lipids, lipoproteins, and apolipoproteins and apolipoprotein E genotype. Journal of Lipid Research 41:1.8121.822

Georges J-L et al. 1996. Family study of lipoprotein lipase gene polymorphisms and plasma triglycerides. Genetic Epidemiology 13:179-182.

Gerdes LU et al. 1996. The apolipoprotein E polymorphism in Greenland Inuit in its global parspective. H uman Genetics 98:546-550.
Goldbourt U, Yaari S \& M edalie JH 1997. I solated low HDL cholesterol as a risk for coronary heart disease mortality: a 21-year follow-up of 8.000 men. Arteriosclerosis, Thrombosis, and Vascular Biology 17:107113.

Gudnason V et al. 1999. Cholesteryl ester transfer protein gene effect on CETP activity and plasma high-density lipoprotein in European populations. European Journal of Clinical Investigation 29:116-128.

Hagberg JM , Wilund KR \& Ferrel RE 2000. APOE gene and gene-environment effects on plasma lipoprotein-lipid levels. Physiological Genomics 4:101-108.

Heller PA, de Faire U, Pederson N, Dahlen G \& M cClearn GE 1993. Genetic and environmental influences on serum lipid levels in twins. N ew England Jouranl of M edicine 328:1.150-1.156.

Hokanson JE \& Austin M A 1996. Plasma triglyceride level is a risk factor for cardiovascular disease independent of high-density liprotein cholesterol level: a meta-analysis of population-based prospective studies. Journal of Cardiovascular Risk 3:213-229.

Hokanson JE 1999. Functional variants in the lipoprotein lipase gene and risk of cardiovascular disease. Current O pinion in Lipidology 10:393-399.

Humphries $S$ et al. 1991. Variation in the low density lipoprotein receptor gene is associated with differences in plasma low density lipoprotein cholesterol levels in young and old normal individuals from Italy. Arteriosclerosis and Thrombosis 11:509-516.

H umphries SE \& Ordovas JM 2001. Genetics and atherosclerosis: broadening the horizon. Atherosclerosis 154:517-519.

International H uman Genome Sequencing Consortium 2001. Initial sequencing and analysis of the human genome. Nature 409:860-921.

Jemaa $R$, Tuzet S, Betoulle $D$, Apfelbaum $M \&$ Fumeron $F$ 1997. Hind III polymorphism of the lipoprotein lipase gene and plasma lipid response to low calorie diet. International Journal of O besity 21:280-283.

Juo S-H H, Han Z, Smith JD, Colangelo L \& Liu K 2001. Promoter polymorphisms of hepatic lipase gene influence $\mathrm{HDL}_{2}$ but not $\mathrm{HDL}_{3}$ in African men: CARDIA study. Journal of Lipid Research 42:258-264.

Kamboh MI, Aston CE \& Hamman RF 1995. The relationship of APOE polymorphism and cholesterol levels in normoglicemic and diabetic subjects in a biethnic population from San Luis Valley, Colorado. Atherosclerosis 112:145-159.

Kammerer CM, Vandeberg JL, H affner SM \& Hixson JE 1996. Apolipoprotein B (apo B) signal peptide length polymorphisms are associated with apo $B$, low density lipoprotein cholesterol, and glucose levels in M exican Americans. Atherosclerosis 120:37-45.

Kark JD et al. 2000. Taq1B CETP polymorphism, plasma CETP, lipoproteins, apolipoproteins and sex differences in a J ewish population sample characterized by low HDL-cholesterol. Atherosclerosis 151:509-518.

Khoury MJ 1997. Relationship between medical genetics and public health: changing the paradigm of disease prevention and the definition of a genetic disease. American Journal of M edical Genetics 17:289-291.

Ko Y-L et al. 1997. Interaction between obesity and genetic polymorphisms in the apolipoprotein CIII gene and lipoprotein lipase gene on the risk of hypertrigliceridemia in Chinese. Human Genetics 100: 327-333. 
Korhonen T, Savolainen MJ \& Kesäniemi YA 1999. Variation of apolipoprotein $B$ as a possible cause of decreased low density lipoprotein clearance and hypercholesterolemia. Atherosclerosis 146:1-10.

M achado M O, Hirata M H, Bertolami M C \& H irata RDC 2001. Apo B gene haplotype is associated with lipid profile of higher risk of coronary heart disease in caucasian Brazilian men. Journal of Clinical Laboratory Analysis 15:19-24.

Mahley RW et al. 1995. Turkish Heart Study: lipids, lipoproteins, and apolipoproteins. Journal of Lipid Research 36:839-859.

M aron DJ, Ridker PM \& Pearson TA 1998. Risk factors and the prevention of coronary heart disease, pp.1.175-1.195. In Alexander RW, Schlant RC, Fuster $V, O$ 'Rourke RA, Roberts $R$ \& Sonnenblick EH (eds.). Hurst's the Heart. Arteries and Veins. M cGraw Hill, Nova York.

M attevi VS 2000. Análise da influência de polimorfismos do gene do receptor da lipoproténa de baixa densidade sobre níveis lipídicos e obesidade em população brasileira. Dissertação de M estrado. Programa de Pós-Graduação em Genética e Biologia M olecular, UFRGS, Porto Alegre. 109 pp.

M urray CJL \& Lopez AD 1997. Mortality by cause for eight regions of the world: global burden of disease study, The Lancet 349:1.269-1.276.

NCEP 2001. Executive summary of the third report of the $N$ ational Cholesterol Education Program (NCEP) expert panel on detection, evaluation, and treatment of high blood cholesterol in adults (Adult Treatment Panel III). J ournal of American M edical Association, 285:2.486-2.496

N ebert DW 1999. Pharmacogenetics and pharmacogenomics: why is this relevant to the clinical geneticist? Clinical Genetics 56:247-258.

Pedersen JC \& Berg K 1990. Gene-gene interaction between the low density lipoprotein receptor and apolipoprotein E loci affects lipid levels. Clinical Genetics 44:214-220.

Razzaghi H; Aston CE; Hamman RF \& Kamboh M I 2000. Genetic screening of the lipoprotein lipase gene for mutations associated with high triglyceride/low HDL-cholesterol levels. Human Genetics 107:257267.
Salazar LA et al. 2000. Pvu II intron 15 polymorphism at the LDL receptor gene is associated with differences in serum lipid concentrations in subjects with low and high risk for coronary artery disease from Brazil. Clinica Chimica Acta 293:75-88.

Sephernia B et al. 1989. Genetic studies of human apoliproteins. X. The effect of the apolipoprotein $E$ polymorphism on quantitative levels of lipoproteins in Nigerian blacks. American Journal of $\mathrm{H}$ uman $\mathrm{Ge}$ netics 45:586-591.

Vohl M-C et al. 1999. Contribution of the cholesteryl ester transfer protein gene Taql $\mathrm{B}$ polymorphism to the reduced plasma HDL-cholesterol levels found in abdominal obese men with the features of the insulin resistance syndrome. International Journal of 0 besity 23:918-925.

WangJ et al. 2000. Common and rare ABCA1 variants affecting plasma HDL cholesterol. Arteriosclerosis, Thrombosis, and Vascular Biology 20:1.983-1.989.

Wang $X L$, Badenhop R, H umphrey KE \& Wilcken DE 1996. N ew M spl polymorphism at +83 bp of the human apolipoprotein Al gene: association with increased circulating high density lipoprotein cholesterol levels. Genetic Epidemiology 13:1-10.

Wang XL, Badenhop RB, Sim AS \& Wilcken DEL 1998. The effect of transcription efficiency of the apolipoprotein AI gene of DNA variants at the 5'untranslated region. International Journal of Clinical and Laboratory Research 28:235-241.

Wilttrup HH, Tybjærg-Hansen A \& N ordestgaard BG 1999. Lipoprotein lipase mutations, plasma lipids and lipoproteins, and risk of ischemic heart disease a meta-analysis. Circulation 99:2.901-2.907.

Winkelman BR \& Hager J 2000. Genetic variation in coronary heart disease and myocardial infarction methodological overview and clinical evidence. Pharmacogenomics 1:73-94.

Zambon A, Deeb SS, Brown G, H okanson JE \& Brunzell JD 2001. Common hepatic lipase gene promoter variant determines clinical response to intensive lipid-lowering treatment. Circulation 103:792-798. 